\title{
Fixed point of nonlinear contractions in modular spaces
}

Rudong Chen ${ }^{*}$ and Xue Wang

"Correspondence: tjcrd@yahoo.com.cn

Department of Mathematics, Tianjin Polytechnic University, Tianjin, 300387, China

\begin{abstract}
Razani et al. studied the fixed points of nonlinear and asymptotic contractions in the modular space in 2007. In this paper, we generalize the kind of nonlinear contraction that is the result of Razani et al. (Abstr. Appl. Anal. 2007:40575, 2007) and prove the existence and uniqueness of fixed points for the generalized nonlinear contractions in modular spaces.
\end{abstract}

Keywords: nonlinear contractions; fixed points; modular spaces

\section{Introduction}

The notion of modular spaces, as a generalization of metric spaces, was introduced by Nakano [1] in 1950 in connection with the theory of order spaces and redefined and generalized by Musielak and Orlicz [2] in 1959. These spaces were developed following the successful theory of Orlicz spaces, which replaces the particular integral form of the nonlinear functional, which controls the growth of members of the space, by an abstractly given functional with some good properties. The monographic exposition of the theory of Orlicz spaces may be found in the book of Krasnosel'skii and Rutickii [3]. For a current review of the theory of Musielak-Orlicz spaces and modular spaces, the reader is referred to the books of Musielak [2] and Kozlowski [4].

Fixed point theorems in modular spaces, generalizing the classical Banach fixed point theorem in metric spaces, have been studied extensively. In 2007, Razani et al. [5] studied some fixed points of nonlinear and asymptotic contractions in the modular spaces. In addition, quasi-contraction mappings in modular spaces without $\Delta_{2}$-condition were considered by Khamsi [6] in 2008. In 2011, Kuaket and Kumam [7] proved the existence of fixed points of asymptotic pointwise contractions in modular spaces. Recently, we proved the fixed points of asymptotic pointwise nonexpansive mappings in modular spaces [8].

In this paper, we introduce the notions of nonlinear contractions in modular spaces and establish their fixed points theorems in modular spaces.

\section{Preliminaries}

Definition 2.1 Let $X$ be an arbitrary vector space over $K(=R$ or $C)$.

(a) A functional $\rho: X \rightarrow[0, \infty]$ is called modular if

(i) $\rho(x)=0$ if and only if $x=0$;

(ii) $\rho(\alpha x)=\rho(x)$ for $\alpha \in K$ with $|\alpha|=1$, for all $x \in X$;

(iii) $\rho(\alpha x+\beta y) \leq \rho(x)+\rho(y)$ if $\alpha, \beta \geq 0$, for all $x, y \in X$.

๑ 2013 Chen and Wang; licensee Springer. This is an Open Access article distributed under the terms of the Creative Commons Attribution License (http://creativecommons.org/licenses/by/2.0), which permits unrestricted use, distribution, and reproduction in any medium, provided the original work is properly cited. 
If (iii) is replaced by

(iii') $\rho(\alpha x+\beta y) \leq \alpha \rho(x)+\beta \rho(y)$, for $\alpha, \beta \geq 0, \alpha+\beta=1$, for all $x, y \in X$,

then the modular $\rho$ is called convex modular.

(b) A modular $\rho$ defines a corresponding modular space, i.e., the space $X_{\rho}$ given by

$$
X_{\rho}=\{x \in X \mid \rho(\alpha x) \rightarrow 0 \text { as } \alpha \rightarrow 0\} .
$$

Remark 2.2 Note that $\rho$ is an increasing function. Suppose $0<a<b$. Then, property (iii) with $y=0$ shows that $\rho(a x)=\rho\left(\frac{a}{b}(b x)\right) \leq \rho(b x)$.

Definition 2.3 Let $X_{\rho}$ be a modular space.

(a) A sequence $\left\{x_{n}\right\} \subset X_{\rho}$ is said to be $\rho$-convergent to $x \in X_{\rho}$, and write $x_{n} \stackrel{\rho}{\longrightarrow} x$ if $\rho\left(x_{n}-x\right) \rightarrow 0$ as $n \rightarrow \infty$.

(b) A sequence $\left\{x_{n}\right\}$ is called $\rho$-Cauchy whenever $\rho\left(x_{n}-x_{m}\right) \rightarrow 0$ as $n, m \rightarrow \infty$.

(c) $X_{\rho}$ is called $\rho$-complete if any $\rho$-Cauchy sequence is $\rho$-convergent.

(d) A subset $B \subset X_{\rho}$ is called $\rho$-closed if for any sequence $\left\{x_{n}\right\} \subset B \rho$-convergent to $x \in X_{\rho}$, we have $x \in B$.

(e) A $\rho$-closed subset $B \subset X_{\rho}$ is called $\rho$-compact if any sequence $\left\{x_{n}\right\} \subset B$ has a $\rho$-convergent subsequence.

(f) $\rho$ is said to satisfy the $\Delta_{2}$-condition if $\rho\left(2 x_{n}\right) \rightarrow 0$ whenever $\rho\left(x_{n}\right) \rightarrow 0$ as $n \rightarrow \infty$.

(g) We say that $\rho$ has the Fâtou property if $\rho(x-y) \leq \liminf _{n \rightarrow \infty} \rho\left(x_{n}-y_{n}\right)$, whenever $x_{n} \stackrel{\rho}{\longrightarrow} x$ and $y_{n} \stackrel{\rho}{\longrightarrow} y$ as $n \rightarrow \infty$.

(h) A subset $B \subset X_{\rho}$ is said to be $\rho$-bounded if

$$
\operatorname{diam}_{\rho}(B)<\infty
$$

where $\operatorname{diam}_{\rho}(B)=\sup \{\rho(x-y) ; x, y \in B\}$ is called the $\rho$-diameter of $B$.

(i) Define the $\rho$-distance between $x \in X_{\rho}$ and $B \subset X_{\rho}$ as

$$
\operatorname{dis}_{\rho}(x, B)=\inf \{\rho(x-y) ; y \in B\} .
$$

(j) Define the $\rho$-ball, $B_{\rho}(x, r)$, centered at $x \in X_{\rho}$ with radius $r$ as

$$
B_{\rho}(x, r)=\left\{y \in X_{\rho} ; \rho(x-y) \leq r\right\} .
$$

Theorem 2.4 [5, RNMP] Let $X_{\rho}$ be a $\rho$-complete modular space, where $\rho$ satisfies the $\Delta_{2}$-condition. Assume that $\psi: R^{+} \rightarrow[0, \infty)$ is an increasing and upper semi-continuous function satisfying

$$
\psi(t)<t, \quad \forall t>0 .
$$

Let $B$ be a $\rho$-closed subset of $X_{\rho}$, and let $T: B \rightarrow B$ be a mapping such that there exist $c, l \in R^{+}$with $c>l$,

$$
\rho(c(T x-T y)) \leq \psi(\rho(l(x-y)))
$$


The Banach contraction mapping principle shows the existence and uniqueness of a fixed point in a complete metric space; this has been generalized by many mathematicians such as Arandelovic [9], Edelstein [10], Ciric [11], Rakotch [12], Reich [13], Kirk [14], and so forth. In addition, Boyd and Wong [15] studied mappings, which are nonlinear contractions in the metric space. It is necessary to mention that the applications of contraction, generalized contraction principle for self-mappings, and the applications of nonlinear contractions are well known. In the next section, we will prove the existence fixed points theorems for nonlinear contractions in modular space.

\section{Fixed points of nonlinear contractions}

In the sequel, we assume that $\psi: R^{+} \rightarrow[0, \infty)$ is an increasing and upper semi-continuous function satisfying

$$
\left\{\begin{array}{l}
\psi(t)<t, \quad \forall t>0, \\
\psi(0)=0 .
\end{array}\right.
$$

Lemma 3.1 [16] Let $t>0, \psi(t)<t$ if and only if $\lim _{n} \psi^{n}(t)=0$, where $\psi^{n}$ denotes the $n$-times repeated composition of $\psi$ with itself.

Theorem 3.2 Let $X_{\rho}$ be a $\rho$-complete modular space, where $\rho$ satisfies the $\Delta_{2}$-condition. Let $\psi$ be as in the previous definition, let $C$ be a $\rho$-closed subset of $X_{\rho}$, and let $T: C \rightarrow C$ be a mapping such that there exist $c, l \in R^{+}$with $c>l$,

$$
\begin{aligned}
\rho(c(T x-T y)) \leq & \psi(\max \{\rho(l(x-y)), \rho(l(x-T x)), \rho(l(y-T y)), \\
& \left.\left.\frac{1}{2}[\rho(l(x-T y))+\rho(l(y-T x))]\right\}\right)
\end{aligned}
$$

for all $x, y \in C$. Then $T$ has a unique fixed point.

Proof Let $x \in X_{\rho}$. At first, we show that the sequence $\left\{\rho\left(c\left(T^{n} x-T^{n-1} x\right)\right)\right\}$ converges to 0 .

For $n \in N$, we have

$$
\begin{aligned}
& \rho\left(c\left(T^{n} x-T^{n-1} x\right)\right) \\
& \quad \leq \psi\left(\operatorname { m a x } \left\{\rho\left(l\left(T^{n-1} x-T^{n-2} x\right)\right), \rho\left(l\left(T^{n-1} x-T^{n} x\right)\right),\right.\right. \\
& \quad \rho\left(l\left(T^{n-2} x-T^{n-1} x\right)\right), \frac{1}{2}\left[\rho\left(l\left(T^{n-1} x-T^{n-1} x\right)\right)\right. \\
& \left.\left.\left.\quad+\rho\left(l\left(T^{n-2} x-T^{n} x\right)\right)\right]\right\}\right) .
\end{aligned}
$$

Note that

$$
\begin{aligned}
& \rho\left(l\left(T^{n-2} x-T^{n} x\right)\right) \\
& \quad=\rho\left(l\left(T^{n} x-T^{n-1} x+T^{n-1} x-T^{n-2} x\right)\right) \\
& \quad \leq \rho\left(l\left(T^{n} x-T^{n-1} x\right)\right)+\rho\left(l\left(T^{n-1} x-T^{n-2} x\right)\right) .
\end{aligned}
$$


By (3.3) and (3.4), therefore, we have

$$
\begin{aligned}
\rho\left(c\left(T^{n} x-T^{n-1} x\right)\right) & \leq \psi\left(\rho\left(l\left(T^{n-1} x-T^{n-2} x\right)\right)\right) \\
& <\rho\left(l\left(T^{n-1} x-T^{n-2} x\right)\right)<\rho\left(c\left(T^{n-1} x-T^{n-2} x\right)\right) .
\end{aligned}
$$

Consequently, $\rho\left(c\left(T^{n} x-T^{n-1} x\right)\right)$ is decreasing and bounded from below $(\rho(x) \geq 0)$.

Therefore, $\rho\left(c\left(T^{n} x-T^{n-1} x\right)\right)$ converges to $a$.

Now, if $a \neq 0$

$$
\begin{aligned}
a & =\lim _{n \rightarrow \infty} \rho\left(c\left(T^{n} x-T^{n-1} x\right)\right) \leq \lim _{n \rightarrow \infty} \psi\left(\rho\left(l\left(T^{n-1} x-T^{n-2} x\right)\right)\right) \\
& \leq \lim _{n \rightarrow \infty} \psi\left(\rho\left(c\left(T^{n-1} x-T^{n-2} x\right)\right)\right),
\end{aligned}
$$

then

$$
a \leq \psi(a)
$$

which is a contradiction, therefore, $a=0$.

Now, we show that $\left\{T^{n} x\right\}$ is a $\rho$-Cauchy sequence for $x \in X_{\rho}$. Suppose that $\left\{l T^{n} x\right\}$ is not a $\rho$-Cauchy sequence. Then, there are an $\varepsilon>0$ and sequences of integers $\left\{m_{k}\right\},\left\{n_{k}\right\}$, with $m_{k}>n_{k} \geq k$, and such that

$$
d_{k}=\rho\left(l\left(T^{m_{k}} x-T^{n_{k}} x\right)\right) \geq \varepsilon \quad \text { for } k=1,2, \ldots
$$

we can assume that

$$
\rho\left(l\left(T^{m_{k}-1} x-T^{n_{k}} x\right)\right)<\varepsilon .
$$

Let $m_{k}$ be the smallest number exceeding $n_{k}$, for which (3.8) holds, and

$$
\Sigma_{k}=\left\{m \in N \mid \exists n_{k} \in N ; \rho\left(l\left(T^{m} x-T^{n_{k}} x\right)\right) \geq \varepsilon, m>n_{k} \geq k\right\} .
$$

Obviously, $\Sigma_{k} \neq \emptyset$ and since $\Sigma_{k} \subset N$, then by Well Ordering Principle, the minimum element of $\Sigma_{k}$ is denoted by $m_{k}$, and clearly (3.9) holds.

Now, we assume that $\alpha_{0} \in R^{+}$and $\frac{l}{c}+\frac{1}{\alpha_{0}}=1$, then we have

$$
\begin{aligned}
d_{k} & =\rho\left(l\left(T^{m_{k}} x-T^{n_{k}} x\right)\right) \\
& =\rho\left(\frac{l c}{c}\left(T^{m_{k}} x-T^{n_{k}+1} x+T^{n_{k}+1} x-T^{n_{k}} x\right)\right) \\
& \leq \rho\left(c\left(T^{m_{k}} x-T^{n_{k}+1} x\right)\right)+\rho\left(\alpha_{0} l\left(T^{n_{k}+1} x-T^{n_{k}} x\right)\right) \\
& \leq \psi\left(\rho\left(l\left(T^{m_{k}-1} x-T^{n_{k}} x\right)\right)\right)+\rho\left(\alpha_{0} l\left(T^{n_{k}+1} x-T^{n_{k}} x\right)\right) \\
& \leq \rho\left(l\left(T^{m_{k}-1} x-T^{n_{k}} x\right)\right)+\rho\left(\alpha_{0} l\left(T^{n_{k}+1} x-T^{n_{k}} x\right)\right) \\
& \leq \varepsilon+\rho\left(\alpha_{0} l\left(T^{n_{k}+1} x-T^{n_{k}} x\right)\right) .
\end{aligned}
$$


If $k \rightarrow \infty$ and by $\Delta_{2}$-condition, $\rho\left(\alpha_{0} l\left(T^{n_{k}+1} x-T^{n_{k}} x\right)\right) \rightarrow 0$. Hence, $d_{k} \rightarrow \varepsilon$. Now,

$$
\begin{aligned}
d_{k}= & \rho\left(l\left(T^{m_{k}} x-T^{n_{k}} x\right)\right) \\
\leq & \rho\left(c\left(T^{m_{k}+1} x-T^{n_{k}+1} x\right)\right)+\rho\left(2 \alpha_{0} l\left(T^{m_{k}} x-T^{m_{k}+1} x\right)\right)+\rho\left(2 \alpha_{0} l\left(T^{n_{k}+1} x-T^{n_{k}} x\right)\right) \\
\leq & \psi\left(\rho\left(l\left(T^{m_{k}+1} x-T^{n_{k}+1} x\right)\right)\right)+\rho\left(2 \alpha_{0} l\left(T^{m_{k}} x-T^{m_{k}+1} x\right)\right) \\
& +\rho\left(2 \alpha_{0} l\left(T^{n_{k}+1} x-T^{n_{k}} x\right)\right) .
\end{aligned}
$$

Thus, as $k \rightarrow \infty$, we obtain $\varepsilon \leq \psi(\varepsilon)$, which is a contradiction for $\varepsilon>0$. So, $\left\{l T^{n} x\right\}$ is a $\rho$-Cauchy sequence, and by $\Delta_{2}$-condition, $\left\{T^{n} x\right\}$ is a $\rho$-Cauchy sequence, and $X_{\rho}$ is $\rho$ complete, there is a $\omega \in C$ such that $\rho\left(T^{n} x-\omega\right) \rightarrow 0$ as $n \rightarrow \infty$. Now, it is enough to show that $\omega$ is a fixed point of $T$. Indeed,

$$
\begin{aligned}
\rho\left(\frac{c}{2}(T \omega-\omega)\right) & =\rho\left(\frac{c}{2}\left(T \omega-T^{n+1} x\right)+\frac{c}{2}\left(T^{n+1} x-\omega\right)\right) \\
& \leq \rho\left(c\left(T \omega-T^{n+1} x\right)\right)+\rho\left(c\left(T^{n+1} x-\omega\right)\right) \\
& \leq \psi\left(\rho\left(l\left(\omega-T^{n} x\right)\right)\right)+\rho\left(c\left(T^{n+1} x-\omega\right)\right) \\
& \leq \rho\left(c\left(\omega-T^{n} x\right)\right)+\rho\left(c\left(T^{n+1} x-\omega\right)\right) .
\end{aligned}
$$

Since $\rho\left(c\left(\omega-T^{n} x\right)\right)+\rho\left(c\left(T^{n+1} x-\omega\right)\right) \rightarrow 0$ as $n \rightarrow \infty$, therefore, $\rho\left(\frac{c}{2}(T \omega-\omega)\right)=0$ and $T \omega=\omega$.

Next, we prove that $T$ has a unique fixed point. Letting $\omega^{*}$ be another fixed point of $T$, we have

$$
\rho\left(c\left(\omega-\omega^{*}\right)\right)=\rho\left(c\left(T \omega-T \omega^{*}\right)\right) \leq \psi\left(\rho\left(l\left(\omega-\omega^{*}\right)\right)\right) \leq \rho\left(c\left(\omega-\omega^{*}\right)\right),
$$

which implies that $\rho\left(c\left(\omega-\omega^{*}\right)\right)=0$, so $\omega=\omega^{*}$.

The proof is complete.

The next corollary is immediate consequence of Theorem 3.2.

Corollary 3.3 Let $X_{\rho}$ be a $\rho$-complete modular space, where $\rho$ satisfies the $\Delta_{2}$-condition. Let $C$ be a $\rho$-closed subset of $X_{\rho}$, and let $T: C \rightarrow C$ be a mapping such that there exist $c, k, l \in R^{+}$with $c>l$ and $k \in(0,1)$,

$$
\begin{aligned}
\rho(c(T x-T y)) \leq & k \max \{\rho(l(x-y)), \rho(l(x-T x)), \rho(l(y-T y)), \\
& \left.\frac{1}{2}[\rho(l(x-T y))+\rho(l(y-T x))]\right\}
\end{aligned}
$$

for all $x, y \in C$. Then $T$ has a unique fixed point.

Next, we continue to generalize the above consequences.

Firstly, for any $x \in C$, define the orbit

$$
O(x)=\left\{x, T x, T^{2} x, \ldots\right\}
$$


and its $\rho$-diameter by

$$
\delta_{\rho}(x)=\operatorname{diam}(O(x))=\sup \left\{\rho\left(T^{n} x-T^{m} x\right) ; n, m \in\{0\} \cup N\right\}
$$

then, define

$$
\tilde{\delta}_{\rho}(x)=\sup \left\{\rho\left(c\left(T^{n} x-T^{m} x\right)\right) ; n, m \in\{0\} \cup N\right\} .
$$

The following lemmas will be helpful to prove the main result.

Lemma 3.4 Let $X_{\rho}, C, \psi$ be as in the definitions above, let $T: C \rightarrow C$ be a mapping such that there exist $c, l \in R^{+}$with $c>l$,

$$
\begin{gathered}
\rho(c(T x-T y)) \leq \psi \\
\psi(\max \{\rho(l(x-y)), \rho(l(x-T x)), \rho(l(y-T y)), \\
\rho(l(x-T y)), \rho(l(y-T x))\})
\end{gathered}
$$

for all $x, y \in C$. Let $x \in C$ such that $\tilde{\delta}_{\rho}(x)<\infty$. Then for any $n \geq 1$, one has

$$
\tilde{\delta}_{\rho}\left(T^{n} x\right) \leq \psi^{n}\left(\tilde{\delta}_{\rho}(x)\right)
$$

Moreover, one has

$$
\rho\left(c\left(T^{n} x-T^{n+m} x\right)\right) \leq \psi^{n}\left(\tilde{\delta}_{\rho}(x)\right)
$$

for all $n \geq 1$ and $m \in N$.

Proof Letting $n, m \geq 1$, we have

$$
\begin{aligned}
\rho\left(c\left(T^{n} x-T^{m} y\right)\right) \leq & \psi\left(\operatorname { m a x } \left\{\rho\left(l\left(T^{n-1} x-T^{m-1} y\right)\right), \rho\left(l\left(T^{n-1} x-T^{n} x\right)\right),\right.\right. \\
& \left.\left.\rho\left(l\left(T^{m-1} y-T^{m} y\right)\right), \rho\left(l\left(T^{n-1} x-T^{m} y\right)\right), \rho\left(l\left(T^{n} x-T^{m-1} y\right)\right)\right\}\right)
\end{aligned}
$$

for all $x, y \in C$. This obviously implies the following

$$
\tilde{\delta}_{\rho}\left(T^{n} x\right) \leq \psi\left(\tilde{\delta}_{\rho}\left(T^{n-1} x\right)\right)
$$

for any $n \geq 1$.

Hence, for any $n \geq 1$, we have

$$
\tilde{\delta}_{\rho}\left(T^{n} x\right) \leq \psi^{n}\left(\tilde{\delta}_{\rho}(x)\right)
$$

Moreover, for any $n \geq 1$ and $m \in N$, we have

$$
\rho\left(c\left(T^{n} x-T^{n+m} x\right)\right) \leq \tilde{\delta}_{\rho}\left(T^{n} x\right) \leq \psi^{n}\left(\tilde{\delta}_{\rho}(x)\right) .
$$


Lemma 3.5 Let $X_{\rho}, T, C, \psi, x$ be as in the previous lemma, and let $\rho$ satisfy the Fâtou property. Then $\left\{T^{n} x\right\} \rho$-converges to $\omega \in C$. Moreover, one has

$$
\rho\left(c\left(T^{n} x-\omega\right)\right) \leq \psi^{n}\left(\tilde{\delta}_{\rho}(x)\right)
$$

for any $n \geq 1$.

Proof From the previous lemma, it is easy to know that $\left\{T^{n} x\right\}$ is $\rho$-Cauchy. Since $C$ is $\rho$-closed, then there exists $\omega \in C$ such that $\left\{T^{n} x\right\} \stackrel{\rho}{\longrightarrow} \omega$. Since

$$
\rho\left(c\left(T^{n} x-T^{n+m} x\right)\right) \leq \psi^{n}\left(\tilde{\delta}_{\rho}(x)\right)
$$

for any $n \geq 1, m \in N$, and $\rho$ satisfies the Fâtou property, we let $m \rightarrow \infty$ to get

$$
\rho\left(c\left(T^{n} x-\omega\right)\right) \leq \psi^{n}\left(\tilde{\delta}_{\rho}(x)\right) .
$$

Theorem 3.6 Let $X_{\rho}, \rho, T, C, \psi$ be as in Lemma 3.5. Assume that $\omega \in C$ is the $\rho$-limit of $\left\{T^{n} x\right\}$, and $\rho(c(\omega-T \omega))<\infty, \rho(c(x-T \omega))<\infty$. Then $\omega$ is a fixed point of $T$, i.e., $T(\omega)=\omega$. Moreover, if $\omega^{*}$ is another fixed point of $T$ in $C$ such that $\rho\left(c\left(\omega-\omega^{*}\right)\right)<\infty$, then we have $\omega=\omega^{*}$.

Proof We have

$$
\begin{aligned}
\rho(c(T x-T \omega)) \leq & \psi(\max \{\rho(l(x-\omega)), \rho(l(x-T x)), \rho(l(\omega-T \omega)), \\
& \rho(l(x-T \omega)), \rho(l(\omega-T x))\}) .
\end{aligned}
$$

From (3.15) and the previous results, we get

$$
\rho(c(T x-T \omega)) \leq \psi\left(\max \left\{\tilde{\delta}_{\rho}(x), \rho(c(\omega-T \omega)), \rho(c(x-T \omega))\right\}\right) .
$$

Assume that for $n \geq 1$, we have

$$
\rho\left(c\left(T^{n} x-T \omega\right)\right) \leq \max \left\{\psi^{n}\left(\tilde{\delta}_{\rho}(x)\right), \psi(\rho(c(\omega-T \omega))), \psi^{n}(\rho(c(x-T \omega)))\right\} .
$$

Then

$$
\begin{aligned}
\rho\left(c\left(T^{n+1} x-T \omega\right)\right) \leq & \psi\left(\operatorname { m a x } \left\{\rho\left(l\left(T^{n} x-\omega\right)\right), \rho\left(l\left(T^{n} x-T^{n+1} x\right)\right), \rho(l(\omega-T \omega)),\right.\right. \\
& \left.\left.\rho\left(l\left(T^{n} x-T \omega\right)\right), \rho\left(l\left(T^{n+1} x-\omega\right)\right)\right\}\right) .
\end{aligned}
$$

Hence

$$
\rho\left(c\left(T^{n+1} x-T \omega\right)\right) \leq \psi\left(\max \left\{\psi^{n}\left(\tilde{\delta}_{\rho}(x)\right), \rho(c(\omega-T \omega)), \rho\left(c\left(T^{n} x-T \omega\right)\right)\right\}\right) .
$$

Using our previous assumption, we get

$$
\rho\left(c\left(T^{n+1} x-T \omega\right)\right) \leq \max \left\{\psi^{n+1}\left(\tilde{\delta}_{\rho}(x)\right), \psi(\rho(c(\omega-T \omega))), \psi^{n+1}(\rho(c(x-T \omega)))\right\} .
$$


So, by induction, we have

$$
\rho\left(c\left(T^{n} x-T \omega\right)\right) \leq \max \left\{\psi^{n}\left(\tilde{\delta}_{\rho}(x)\right), \psi(\rho(c(\omega-T \omega))), \psi^{n}(\rho(c(x-T \omega)))\right\}
$$

for any $n \geq 1$. Therefore, we have

$$
\limsup _{n \rightarrow \infty} \rho\left(c\left(T^{n} x-T \omega\right)\right) \leq \psi(\rho(c(\omega-T \omega))) .
$$

Using the Fâtou property, satisfied by $\rho$, we get

$$
\rho(c(\omega-T \omega)) \leq \liminf _{n \rightarrow \infty} \rho\left(c\left(T^{n} x-T \omega\right)\right) \leq \psi(\rho(c(\omega-T \omega))),
$$

so, we get $\rho(c(\omega-T \omega))=0$, i.e., $T(\omega)=\omega$.

Let $\omega^{*}$ be another fixed point of $T$ such that $\rho\left(c\left(\omega-\omega^{*}\right)\right)<\infty$. Then we get

$$
\rho\left(c\left(\omega-\omega^{*}\right)\right)=\rho\left(c\left(T \omega-T \omega^{*}\right)\right) \leq \psi\left(\rho\left(c\left(\omega-\omega^{*}\right)\right)\right),
$$

which implies $\rho\left(c\left(\omega-\omega^{*}\right)\right)=0$, i.e., $\omega=\omega^{*}$.

We complete the proof of our theorem.

The following corollary is an immediate consequence of Theorem 3.6.

Corollary 3.7 [6] Let $X_{\rho}$ be a $\rho$-complete modular space, where $\rho$ satisfies the Fâtou property. Let $C$ be a $\rho$-closed subset of $X_{\rho}$, and let $T: C \rightarrow C$ be a mapping such that there exist $c, k, l \in R^{+}$with $c>l$ and $k \in(0,1)$,

$$
\begin{gathered}
\rho(c(T x-T y)) \leq k \max \{\rho(l(x-y)), \rho(l(x-T x)), \rho(l(y-T y)), \\
\rho(l(x-T y)), \rho(l(y-T x))\}
\end{gathered}
$$

for all $x, y \in C$. Assume that $\omega \in C$ is the $\rho$-limit of $\left\{T^{n} x\right\}$, and $\rho(c(\omega-T \omega))<\infty, \rho(c(x-$ $T \omega))<\infty$. Then $\omega$ is a fixed point of $T$, i.e., $T(\omega)=\omega$. Moreover, if $\omega^{*}$ is another fixed point of $T$ in $C$ such that $\rho\left(c\left(\omega-\omega^{*}\right)\right)<\infty$, then we have $\omega=\omega^{*}$.

\section{Competing interests}

The authors declare that they have no competing interests.

\section{Authors' contributions}

$\mathrm{RC}$ was introduce and design the question, proof of theorem by XW, RC finally to revise, finalized, submit it to journal.

\section{Acknowledgements}

This research was supported by the NSFC Grant No. 11071279.

Received: 31 August 2012 Accepted: 30 July 2013 Published: 22 August 2013

\section{References}

1. Nakano, H: Modulared Semi-Ordered Spaces. Maruzen Co., Tokyo (1950)

2. Musielak, J, Orlicz, W: On modular space. Stud. Math. 18, 49-65 (1959)

3. Krasnoselskii, MA, Rutickii, YB: Convex Functions and Orlicz Spaces. Fizmatgiz, Moscow (1961) (in Russian). English translation: Noordhoff, Groningen

4. Koslowski, WM: Modular Function Spaces. Dekker, New York (1988) 
5. Razani, A, Nabizadeh, E, Mohamadi, MB, Pour, SH: Fixed points of nonlinear and asymptotic contraction in the modular spaces. Abstr. Appl. Anal. 2007, Article ID 40575 (2007)

6. Khamsi, MA: Quasi-contraction mappings in modular spaces without $\Delta_{2}$-condition. Fixed Point Theory Appl. 2008 Article ID 916187 (2008)

7. Kuaket, K, Kumam, P: Fixed points of asymptotic pointwise contractions in modular spaces. Appl. Math. Lett. 24 1795-1798 (2011)

8. Wang, X, Chen, Y, Chen, R: Fixed points of asymptotic pointwise nonexpansive mappings in modular spaces. J. Appl. Math. 2012, Article ID 319394 (2012)

9. Arandelovic, ID: On a fixed point theorem of Kirk. J. Math. Anal. Appl. 301(2), 384-385 (2005)

10. Edelstein, M: On fixed and periodic points under contractive mappings. J. Lond. Math. Soc. 37(1), 74-79 (1962)

11. Ciric, LB: A generalization of Banach's contraction principle. Proc. Am. Math. Soc. 45(2), 267-273 (1974)

12. Rakotch, E: A note on contractive mappings. Proc. Am. Math. Soc. 13(3), 459-465 (1962)

13. Reich, S: Fixed points of contractive functions. Boll. Unione Mat. Ital. 5(4), 26-42 (1972)

14. Kirk, WA: Contraction mappings and extensions. In: Kirk, WA, Sims, B (eds.) Handbook of Metric Fixed Point Theory, pp. 1-34. Kluwer Academic, Dordrecht (2001)

15. Boyd, DW, Wong, JSW: On nonlinear contractions. Proc. Am. Math. Soc. 20(2), 458-464 (1969)

16. Singh, SP, Meade, BA: On common fixed point theorem. Bull. Aust. Math. Soc. 16, 49-53 (1977)

doi:10.1186/1029-242X-2013-399

Cite this article as: Chen and Wang: Fixed point of nonlinear contractions in modular spaces. Journal of Inequalities and Applications 2013 2013:399.

\section{Submit your manuscript to a SpringerOpen ${ }^{\circ}$ journal and benefit from:}

- Convenient online submission

- Rigorous peer review

- Immediate publication on acceptance

- Open access: articles freely available online

- High visibility within the field

- Retaining the copyright to your article 